\title{
A fascial frustum valve for aortic valve replacement
}

\author{
A. K. YATES \\ Department of Thoracic Surgery, Guy's Hospital, London, S.E.1
}

The promising qualities of autologous fascia lata in heart valve replacement have resulted in a search for improved methods of fascial heart valve manufacture. This paper describes a simply made and inserted and reliably competent unsupported fascial valve for aortic valve replacement.

\section{TECHNICAL DETAILS}

After consideration of detailed measurements of normal aortic valves the shape of the fascial component adopted was a frustum (horizontal section of a cone). This affords sufficient tissue for natural cusp configuration without shaping, overcoming the difficulty of cusp disproportion and allowing natural variation in individual cusp size. In the open position the valve's cross-sectional area progressively increases from its base at the aortic root to its distal attachment.

Each aortic root size requires a different frustum which has to be calculated.

Considering Fig. 1, $\mathrm{r}$ is the radius of the respective aortic root and $\mathbf{R}$ is the maximum radius of the aorta below the sinus ridges. The value of $R$ is a mean of measurements directly taken of a series of hearts. This value was found to have significant correlation in different hearts of the same aortic root size, except in cases of Marfan's disease and syphilitic aortitis.

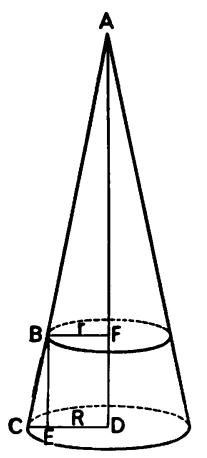

FIG. 1

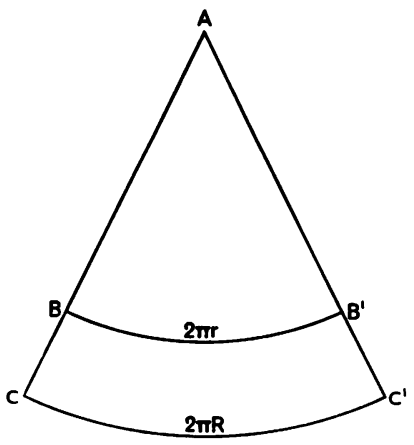

FIG. 2
FIG. 1. Relationship of frustum to cone.

FIG. 2. Frustum opened to provide template.
$\mathrm{BC}$ is the mean distance from the aortic root to the top of the commissures measured in a series of hearts for each aortic root size. Five millimetres $\bar{z}$ has been added to this measurement to allow for suturing and possible future fascial shrinkage and $\frac{\partial}{\partial}$ also affords dependable competence.

To fashion the frustum from flat fascia the frustum must be opened to enable a template $\mathrm{BB}^{\prime} \mathrm{C}^{\prime} \mathrm{C}$ to be made (Fig. 2). This template is a section of a circle and to obtain it the radius $A C$ and the angle CAC' $^{\prime}$ must be found.

Considering the cone, in Fig. 1, perpendicular lines dropped from its apex and from point $B \propto$ produce similar right-angled triangles $\mathrm{ABF}$ and $B C E$ as angle CBE and BAF are the same.

Therefore,

$$
\frac{\mathrm{AB}}{\mathrm{BF}}: \frac{\mathrm{BC}}{\mathrm{CE}}
$$

As all are known except $A B$ this can be calculated and the value of $A C$ gained. $A C$ is the radius of the circle of which the template is a section. 3 Therefore, angle $\mathrm{CAC}^{\prime}$ is $360 \times 2 \pi \mathrm{R}$, and the template can be drawn.

This calculation was made for each respective aortic root size and a steel template was made for each after $3 \mathrm{~mm}$ had been added on to its width to allow for suturing (Fig. 3).

The Table gives the resulting figures.

T A B L E

DETAILED MEASUREMENTS OF FRUSTUM TEMPLATES

\begin{tabular}{|c|c|c|c|c|}
\hline \multirow{2}{*}{$\begin{array}{l}\text { Aortic } \\
\text { Ring } \\
\text { (mm) }\end{array}$} & \multirow[b]{2}{*}{ Angle } & \multicolumn{3}{|c|}{ Template (mm) } \\
\hline & & $\begin{array}{c}\text { Short } \\
\text { Circumf. }\end{array}$ & $\underset{\text { Circumf. }}{\text { Long }}$ & Depth \\
\hline $\begin{array}{l}16 \\
18 \\
20 \\
22 \\
24 \\
26 \\
28 \\
30\end{array}$ & $\begin{array}{l}62^{\circ} \\
60^{\circ} \\
57^{\circ} \\
55^{\circ} \\
52^{\circ} \\
51^{\circ} \\
50^{\circ} \\
48^{\circ}\end{array}$ & $\begin{array}{l}53 \\
60 \\
65 \\
72 \\
78 \\
85 \\
91 \\
97\end{array}$ & $\begin{array}{r}78 \\
85 \\
91 \\
97 \\
103 \\
110 \\
116 \\
122\end{array}$ & $\begin{array}{l}23 \\
24 \\
25 \\
26 \\
27 \\
28 \\
29 \\
30\end{array}$ \\
\hline
\end{tabular}




\section{SURGICAL TECHNIQUE}

The heart is exposed by a sternal-splitting incision and, at the same time, a suitable strip of fascia lata is removed from the thigh. Cardiopulmonary bypass is then started and as soon as the diseased aortic valve has been excised the aortic root is measured by obturators. The appropriate pattern is selected and the fascia lata is cut to this shape (Fig. 4).

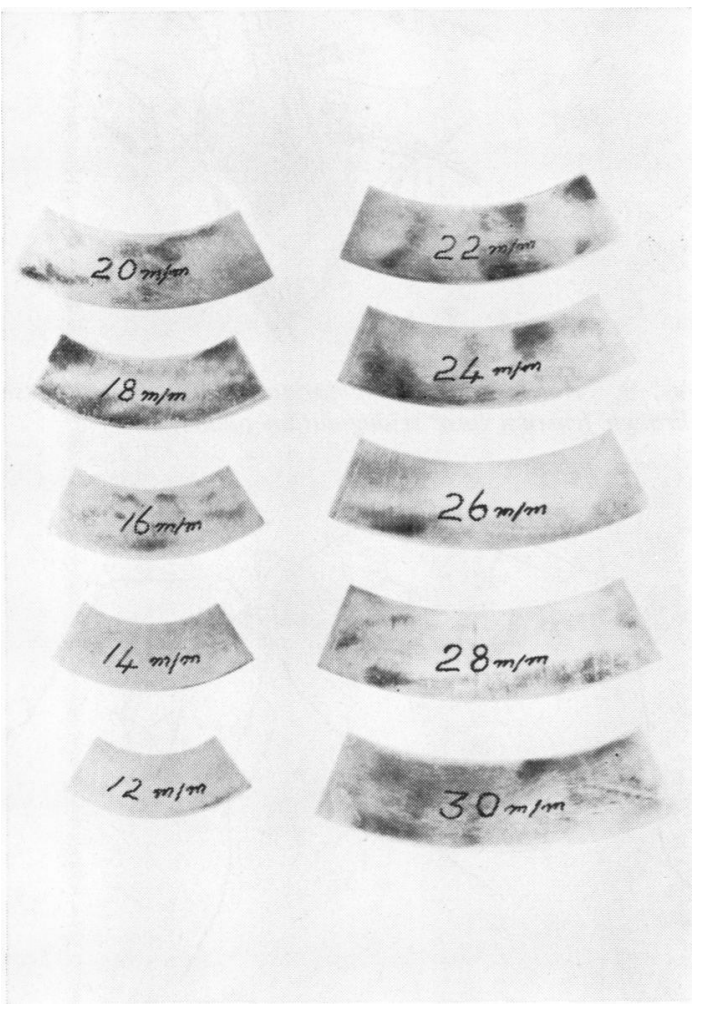

FIG. 3. Stainless steel templates.

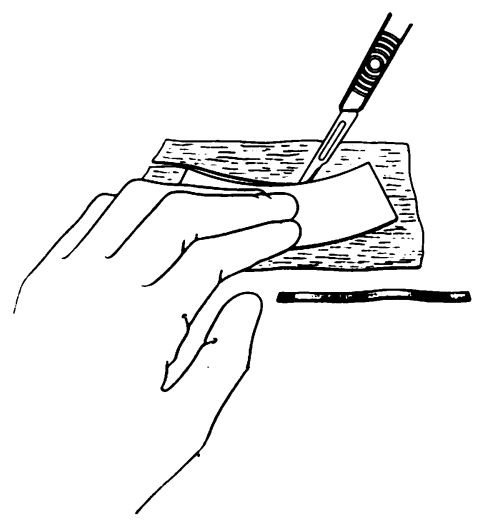

FIG. 4. Fascia lata being cut to shape around template.
A 3-mm strip of Dacron elastic fabric of $0.43 \mathrm{~mm}$ thickness is fixed by a few single sutures to the shorter curved side of the fascia (Fig. 5). The fascia is then sewn into a frustum by suturing its free short edges together by a 3-0 Mersilene double-whip stitch (Fig. 6).

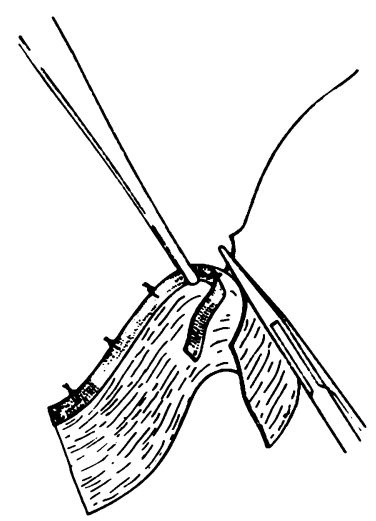

FIG. 5. A 3-mm strip of elastic Dacron being attached to fascia_lata.

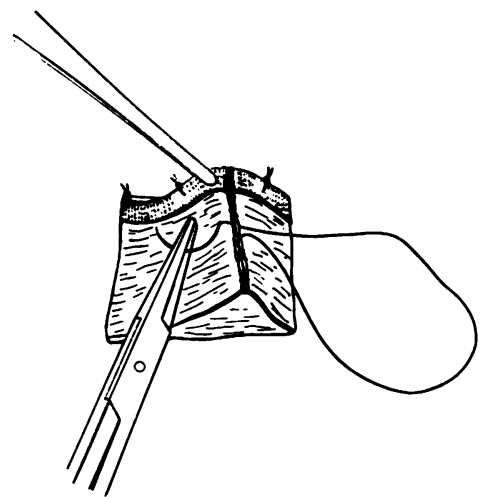

FIG. 6. Fascia lata being sewn to provide a frustum.

The frustum is triangulated at both ends by loose sutures, using a triangulation cone (Fig. 7). This procedure takes approximately 10 minutes and the valve is then ready for insertion.

The aortic root is triangulated by 3-0 Mersilene commissural base sutures (Fig. 8). These sutures are then passed through the triangulation points in the narrow end of the frustum and include the elastic Dacron strip (Fig. 9). The base of the fascial valve can then be sutured horizontally in the aortic root either by the Barratt-Boyes' technique (Figs. 10 to 12) or by using interrupted sutures in the usual method for prosthetic valve insertion. Each suture passes through the fascia and the Dacron strip; this ensures a bloodtight, buttressed suture line. 


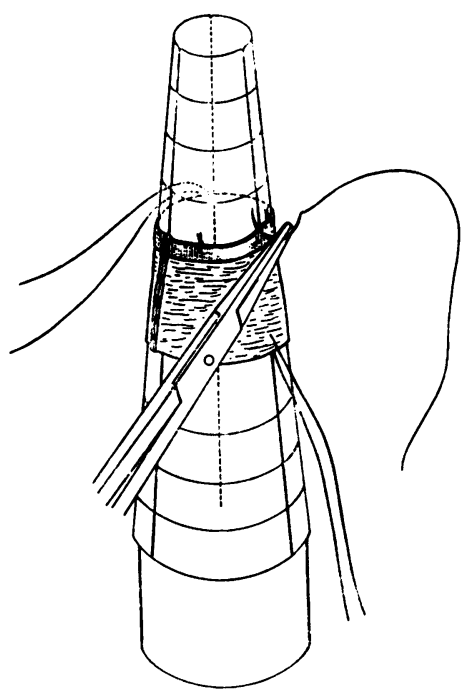

FIG. 7. Triangulation of fascial frustum using a triangulation cone.

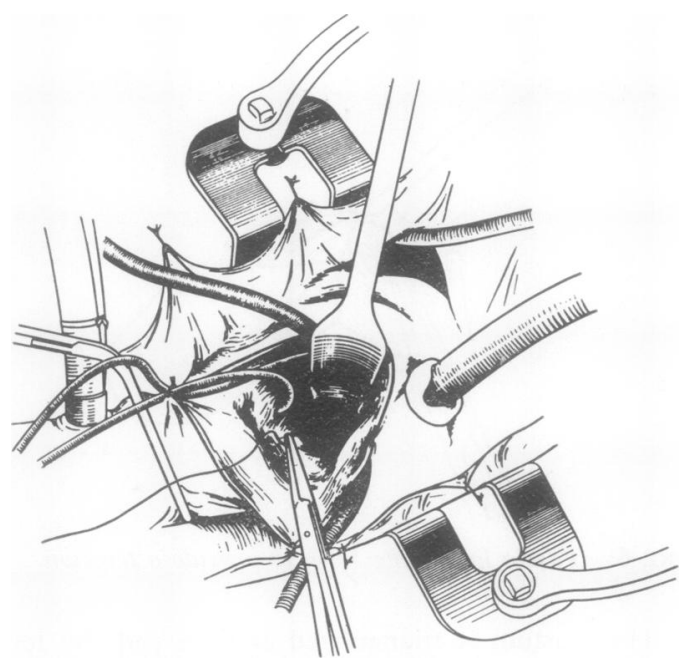

FIG. 8. Triangulation of aortic root.

The final suturing involves fixing the top of the frustum at its three commissural points. As the fascial depth is a little greater than the natural valve depth the point of fixation is above the top of the natural commissure and is found by placing slight traction on the fascial edge at the relevant point. It has been found that only two buttressed sutures are necessary at each point (Figs. 13 and 14). The inner and outer buttresses are 2-mm narrow strips of elastic Dacron (Fig. 15).

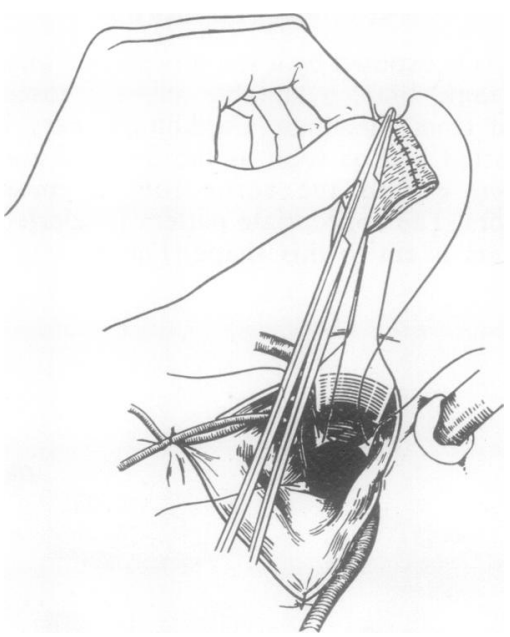

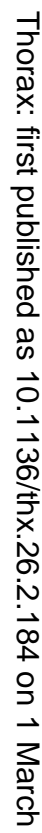

FIG. 9. Aortic root triangulation sutures being passed $\vec{\theta}$ through frustum base triangulation points.

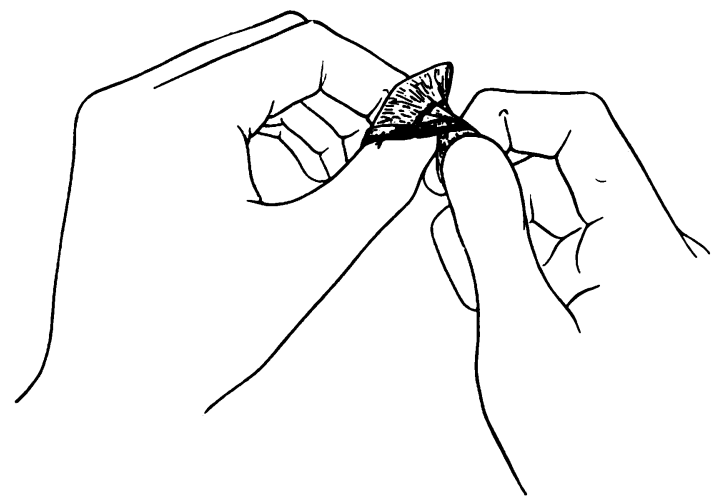

FIG. 10. Fascial frustum being inverted.

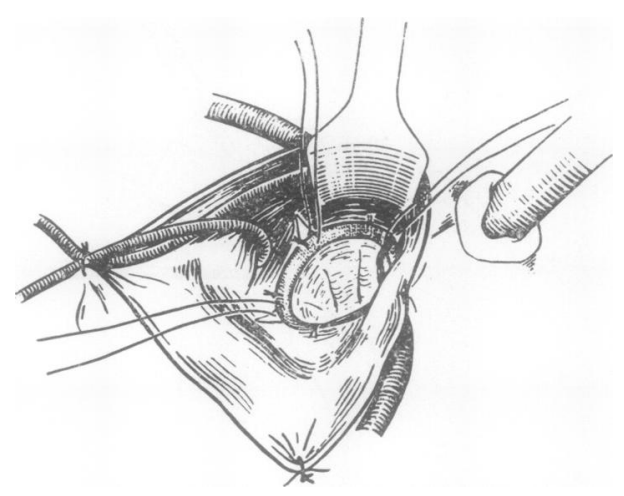

FG. 11. Inverted frustum seated into aortic root. 


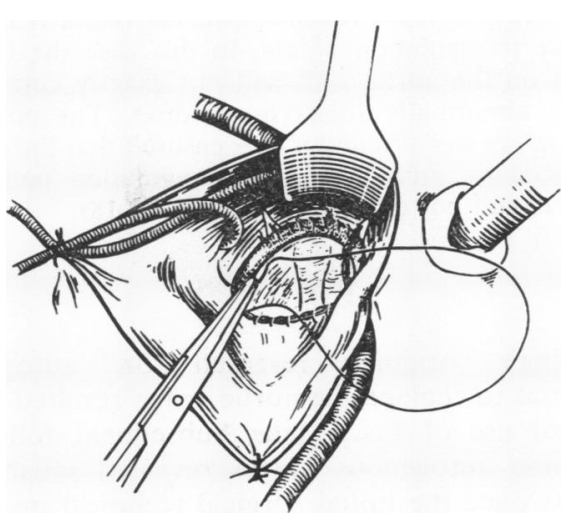

FIG. 12. Inverted frustum sewn into aortic root.

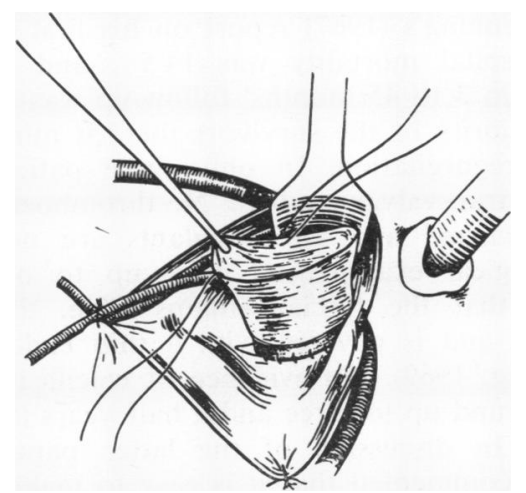

FIG. 13. Fascial frustum pulled up into aorta.

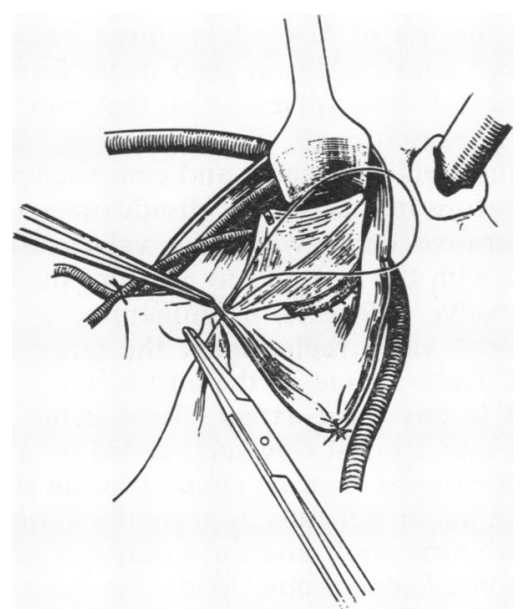

FIG. 14. Fixation of commissure points.

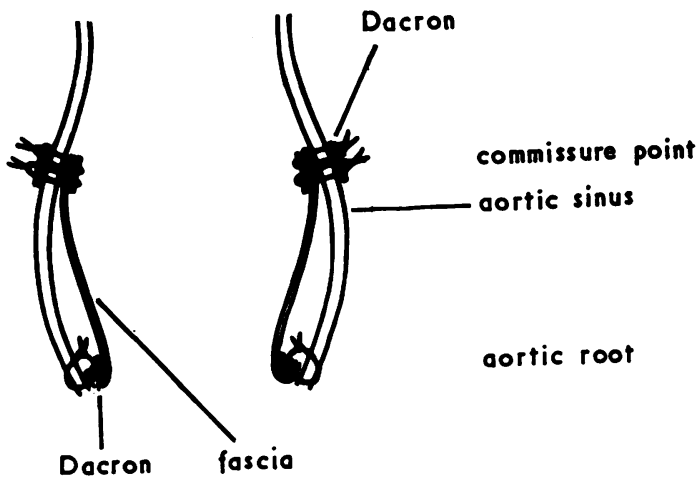

FIG. 15. Cross-sectional representation of valve in situ.

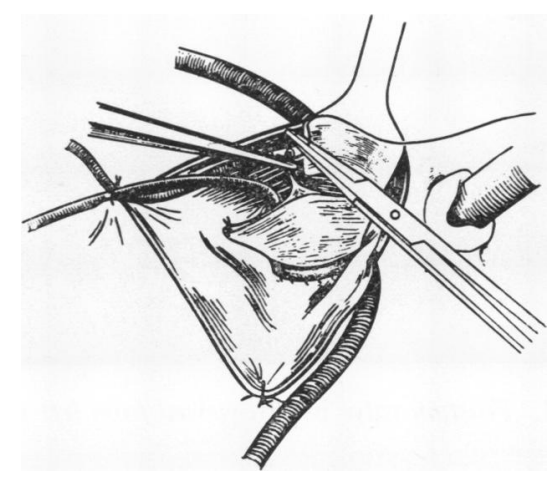

FIG. 16. Final commissure sutures.

The inner strip of Dacron is covered by a single mattress suture of 5-0 Mersilene inserted into the free edges of the fascia at the commissure tops (Fig. 16). The buttressing of sutures as described is important for strength of fixation.

The inherent competency of the valve depends on allowing the cusps to form naturally. In practice the commissural support points can be varied considerably in position around the fascia with no cusp prolapse. It is important that, when sutured, the commissural fixation should be made with slight traction on the fascia.

The normal aortic valve has cusps of differing size, the non-coronary cusp being the deepest. The commissure support points of the fascial frustum can be selected so that they exactly align with the natural commissures. The point on the free fascial edge corresponding with the natural commissure is easily located, being the point at which traction upwards produces a vertical crease in the fascia in line with the excised commissure. These positions will be found to be a millimetre or two from the previously marked triangulation points. In rheumatic valve disease, where the commissures are naturally sited, they should be followed. 


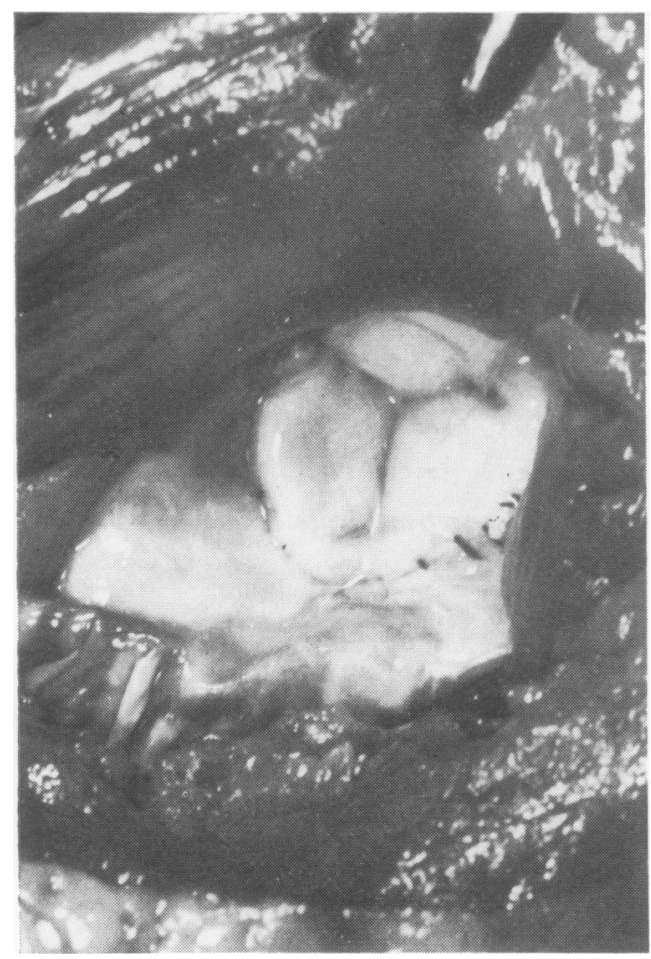

FIG. 17. Frustum valve in situ viewed from left ventricle.

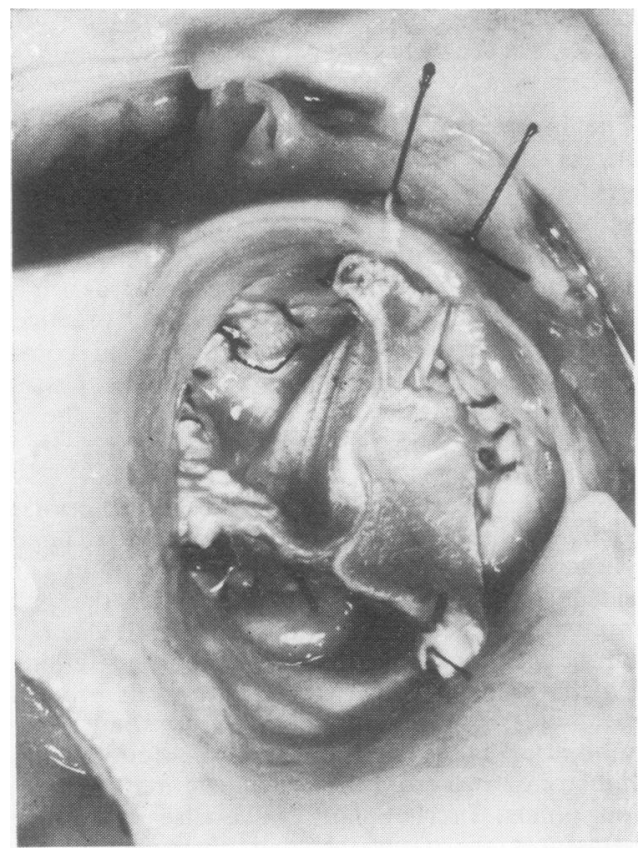

FIG. 18. Frustum valve in situ viewed from above.
In bicuspid valve replacement, the fascia is fixed at its true triangulation points. In this case the fixation? points on the aortic wall will not exactly correspond음 to the abnormally sited commissures. The points of $\overline{\bar{C}}$ fixation are easily found if it is ensured that the creases produced by pulling on the triangulation points are vertical and not oblique (Figs. 17 and 18).

\section{DISCUSSION}

Senning's original research on autogenous $\vec{x}$ material to replace the aortic valve resulted in thei clinical use of fascia lata. Subsequent follow-upiv of these autogenous valves revealed satisfactory $\vec{\infty}$ results once the initial surgical technical problems ${ }^{\star}$ had been overcome. Senning (1966) noted that a definite disadvantage of this operation is the rather long time required to perform it, the per- 3 fusion time being more than two hours.

In Senning's (1967) report on his first 90 cases the hospital mortality was $14.5 \%$ and the late deaths in 3 to 45 months' follow-up was $10 \%$. Inthe majority of the survivors there is minimal or slight regurgitation. In only three patients was there a transvalvar gradient. No thromboembolism has occurred and anticoagulants are not used.๊ Histological examination after up to one year ${ }_{\Omega}^{\mathbb{D}}$ shows that the fascia remains alive, thin, and $\Rightarrow$ flexible and is covered with a thin endothelium 3 (Senning, 1969). No evidence of calcification has been found up to three and a half years (Senning,: 1967). In discussion of the latter paper C. P Bailey commented that it is easy to make a cuspo too large or too small ; either case of dispropor $\frac{0}{x}$ tion will cause incompetence.

Recently (Edwards, Karp, Robillard, and Kerr 1969 ; Ionescu and Ross, 1969), in an attempt tos expand the use of fascia lata, three leaflet valves have been constructed on rigid metal frames. Theo advantage of these valves is that they can be mades at the beginning of the operation, therefore shortening perfusion time, and competency can be tested before insertion. The disadvantages are the intraoperative delay while the valves are made 0 coupled with the inability to measure the required size of valve before its manufacture.

In aortic valve replacement the latter is a sig ${ }_{0}$ nificant disadvantage as the aortic ring cannot be tailored to any great extent. Because the fascia iș sutured into a metal ring surrounded by a Dacrono cuff, which must then be sutured inside the aortio. root, the major difficulty is accommodating a ring into the aortic root, and an unsatisfactorily smalp valve sometimes results. With the Ionescu-Rosstechnique a $22-\mathrm{mm}$ ring can usually be accommodated in large adults, but in women and smalp 


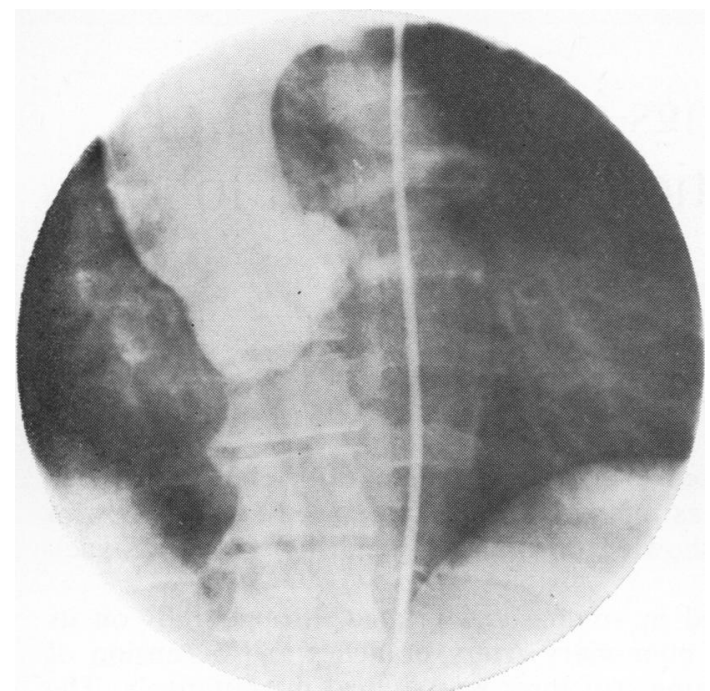

FIG. 19. Antero-posterior postoperative aortogram.

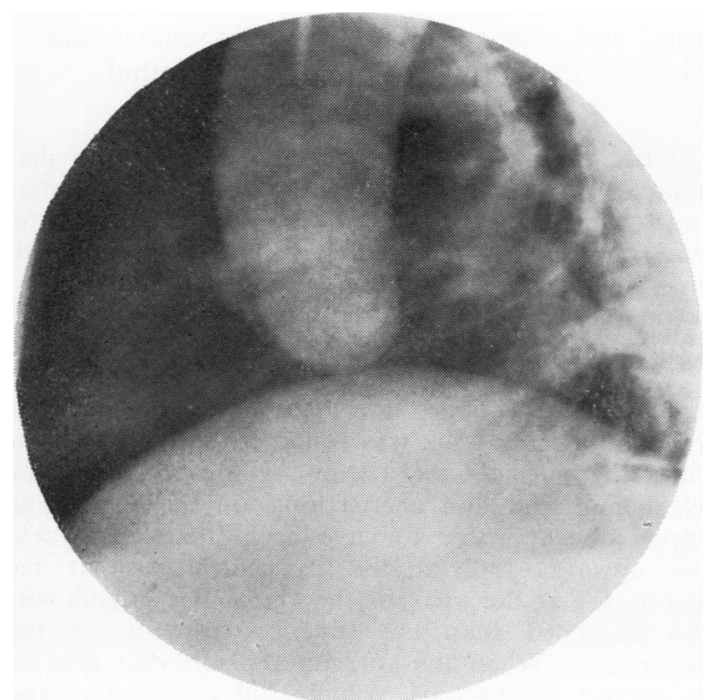

FIG. 20. Lateral postoperative aortogram. males a ring no larger than $20 \mathrm{~mm}$ must be used. The latter valve can exhibit a significant gradient at functional flows.

Consideration of this problem produced the opinion that the unsupported valve has undeniable advantages in the aortic position, including that of non-rigidity. The described valve has been shown to be reliably competent in cadaver hearts, and testing for competency and stability to closing pressures of $180 \mathrm{mmHg}$ have failed to produce tearing or regurgitation. The valve has, to date, been placed in 10 patients with good immediate results. There is no pressure gradient, and good function (Figs. 19 and 20).

This preliminary communication describes a new type of non-supported fascial valve for aortic valve replacement which has the advantages of high-grade competency, ease of manufacture, and quick insertion without incorporation of a metal ring.

There appears to be no reason why this valve should not show the same durable and nonembolic features as other types of fascial valves. The final evaluation will depend on a clinical series with adequate follow-up, which will be reported at a later date.

I am most grateful to Sister V. Wild for her assistance in the detailed valve measurements, and to Miss Gillian Bradford for the illustrations.

\section{REFERENCES}

Edwards, W. S., Karp, R. B., Robillard, D., and Kerr, A. R. (1969). Mitral and aortic valve replacement with fascia lata on a frame. J. thorac. cardiovasc. Surg., 58, 854.

Ionescu, M. I., and Ross, D. N. (1969). Heart-valve replacement with autologous fascia lata. Lancet, 2, 335.

Senning. $\AA$. (1966). Aortic valve replacement with fascia lata. Acta chir. scand., Suppl. 356B, 17. (1967). Fascia lata replacement of aortic valves. J. thorac. cardiovasc. Surg., 54, 465. (1969). Results of fascia lata reconstruction of the aortic valve. J. cardiovasc. Surg. (Torino), Special issue devoted to the XVII Congress of European Society of Cardiovascular Surgery, July 1-3, 1968, p. 28. 